\title{
Comparison of Detection Accuracy and Effect of JPEG2000 Compression on Iris Recognition
}

\author{
Prashant Kapoor \\ M-tech, VLSI, Final year \\ TRUBA Institute-TCST \\ Bhopal
}

\author{
Paresh Rawat, PhD \\ Guide and HOD (ECE) \\ TRUBA Institute-TCST \\ Bhopal
}

\begin{abstract}
In today's digital world, identification based on biometrics has received much attention from research community as well as from industries for security applications. Iris recognition is evolving as one of the most active techniques in biometrics technology accounting to its high reliability for identification and is proved to be most error free means to identify persons. Iris is considered as the reliable biometric feature based on its uniqueness and robustness. To perform iris recognition iris/eye image is captured from numerous person's and these images should be stored in the data base $\&$ retrieved whenever required. Hence there is need of huge databases of iris images. Compression is a unique option available if available storage space is not sufficient for the images. Compression empowers a reduction in the space needed to store these iris images. The aim of this paper is to present the effects of iris image compression on the recognition performance. Usually iris images are 600 times bigger than the Iris Code templates which requires enormous space for storage. It is expected that iris data should be secured, transmitted and embedded in media in the form of images instead of templates. To obtain this objective considering its implications for bandwidth and storage, this paper presents the scheme that combine ROI(region-of-interest) isolation with JPEG 2000 compression at different levels using publicly available database of iris images each in case of two cases of Normalized iris images on with classic Daughman's rubber sheet model and the second one through non-linear Biomechanical model. It is concluded that JPEG 2000 compression gives the better results with iris images normalized with Biomechanical model with minimum impact on recognition performance.
\end{abstract}

\section{General Terms}

Iris Recognition, Biometrics, Authentication systems et. al.

\section{Keywords}

Biometric, Iris Detection, Security purpose, Recognition Performance, compression Matching.

\section{INTRODUCTION}

With the growing demand of biometric application the question arises to store and process the obtained sensor data. The compression of these data may become significant under certain situations due to the large volumes of data involved. The compression technology may be used in two phases of processing in normal biometric recognition methods [1]. In this paper JPEG 2000 schemes is studied for iris image compression allowing very good recognition performance [2].

\section{STORING THE REFERENCE DATA}

In majority of template databases (in which the reference data of the enrolled individuals are secured) only the extracted features which are necessary for the matching phase are stored as compared with originally acquired sensor data. But authorities, regulatory bodies, and international standards organizations state that biometric data should be recorded and stored in a crude form, rather than in post-processed templates. Storage of the original sensor data solves this issue. Data must be stored in abridge and protected form to secure the storage space [1,2].

\section{DATA TRANSMISSION POST SENSOR DATA ACQUISITION}

In discrete biometric systems, since the data acquisition stage is segregated from the feature extraction and matching phase. Hence the sensor data have to be transferred through a network link to designated locations, usually over wireless channels comprising of low bandwidth and high latency. Therefore, a reduction of the amount of data to be transferred is required .This is achieved by compressing the data before transmission [3]. So, the compression of the raw sensor data can be beneficial in many applications, there is need to identify techniques suitable to perform this task in an appropriate manner to maximize the benefit of data reduction, compression techniques have to be applied[1,3]

The present paper, investigate that the crude image data can be compressed without affecting the biometric templates processed from the data and processing an estimation of template and image data sizes with minimum impact on iris recognition performance. Enrolling or storing raw image data also significant which made these databases more robust since to benefit the future improvements in recognition algorithms, merely by enrolling a new the raw data. The directive for, standards bodies like ISO [2] is to avoid incorporating patented techniques into data formats and standards. Storing raw images instead of templates can enhance the size of data manifolds leading to problems like enhanced data transmission and inability to fix the raw data in the provided space. So iris image compression and its impact on recognition performance [2] become critical.

\section{BASIC OF JPEG2000 COMPRESSION TECHNIQUE}

JPEG 2000 was developed by the Joint Photographic Experts Group committee in 2000 [4] with the purpose of enhancements over the original discrete cosine transformbased JPEG standard with a newly designed method. The JPEG 2000 standard provides high flexible modes of compression achieving approximately $30-40 \%$ more compression at a given image quality. This method of compression is based on a Discrete Wavelet Transform (DWT) rather than the Discrete Cosine Transform (DCT) [4]. Block quantization artifacts was a major drawback of JPEG at low bit-rates, JPEG2000 is not suffering with the same because the DCT simply chops cosine waves in box windows with truncation consequences when they are not dense and incomplete. On the other hand the different levels within 
the multi- resolution DWT wavelet [5] allows local areas in each image template to be encoded using separate sub bands of coefficients as needed [5].

The overall superiority of JPEG2000 over other compression algorithms is in terms of image quality particularly marked at the very low bit-rates in proportions to high compression. Different methods exist within JPEG2000 for allocation of template definition allowing different DWT resolution levels in different.

\section{METRICS FOR BIOMETRIC PERFORMANCE}

\subsection{False Acceptance Rate (FAR)}

FAR [6] is the probability that the system wrongly authorizes a non-authorized person, because of incorrectly matching the biometric input with a stored template. The FAR is expressed as a percentage, following the FAR terminology it is the fraction (\%) of invalid inputs which are incorrectly accepted by system.

$\mathrm{FAR}=\frac{\text { impostor scores above threshold }}{\text { all impostor scores }}$

\subsection{False Rejection Rate (FRR)}

FRR [6] is the probability that the system wrongly rejects authorization to an authorized person, accounting to failing to match the biometric input with a stored template. The FRR is expressed as a percentage, following the FRR terminology it is the fraction $(\%)$ of valid inputs which are incorrectly rejected by system.

$\mathrm{FRR}=\frac{\text { genuine scores below threshold }}{\text { all genuine scores }}$

Figure 1 depicts relation between FAR and FRR. As evident from the relationship diagram, it is evident that as threshold limits increases the FAR reduces exponentially and with increasing the threshold the FAR initially remains to a null value but as threshold is increased above a specific value the FRR keeps on increasing exponentially. The specific point at which the FRR becomes equal to the FAR is called as EER [6].

The relationship diagram also dictates that the FAR value is inversely proportional to the FRR value. EER is typically between 0 to 0.1 in value.

\section{FAR - FRR Diagram}

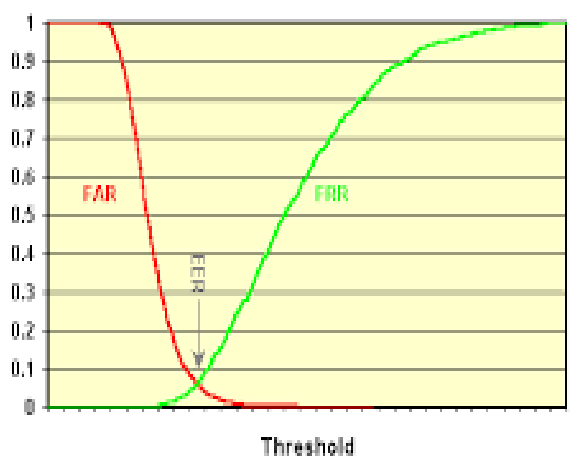

Fig 1: Block diagram of an iris recognition system.

\section{VARIOUS STAGES OF IRIS RECOGNITION}

Figure 2 depicts block diagram for a biometric system of iris recognition

1. Image acquisition: in this stage, a photo is taken from iris [7].

2. Pre-processing: This stage includes edge detection, contrast adjustment and multiplier.

3. Segmentation: This stage includes localization of iris inner and outer boundaries and localization of boundary between iris and eyelids.

4. Normalization: This stage includes transformation from polar to Cartesian coordinates and normalization of iris image [6,7].

5. Feature extraction: This stage includes noise removal from iris image and generating iris code.

6. Classification and matching: This stage includes comparing and matching of iris code with the codes already saved in database as shown in Figure 3.

Iris may have occlusions caused by upper or lower eyelids or eyes may roll left and rightwards

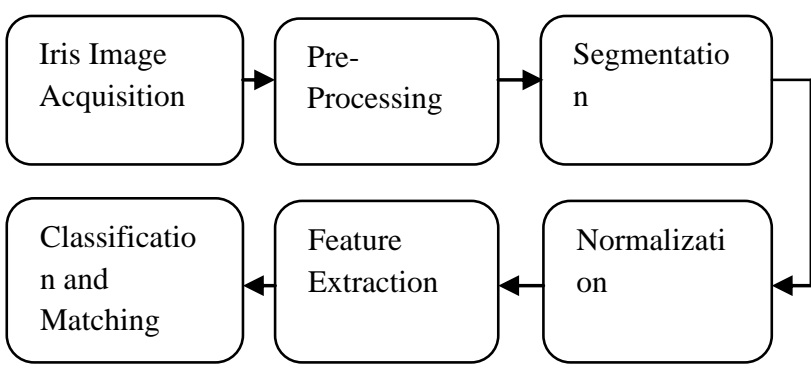

Fig 2: Block diagram of an iris recognition system.

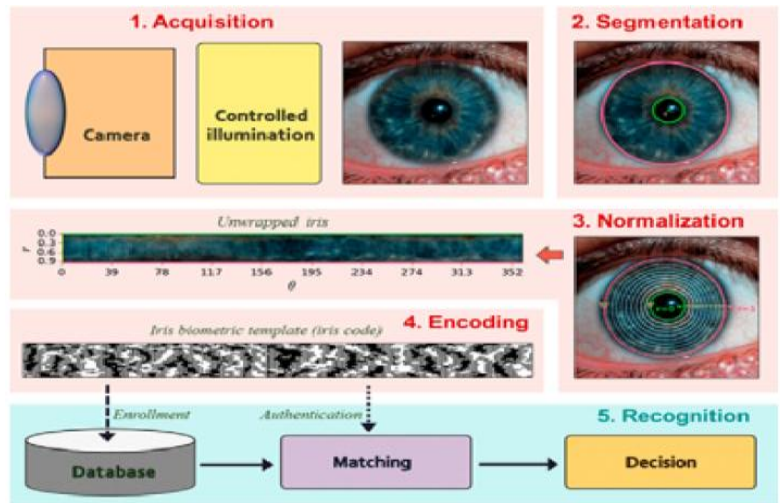

Fig 3: Iris recognition system with stage wise samples

\section{PUPIL/IRIS DILATION}

Pupil dilation is a physical phenomenon that changes the size of the pupil through the optic and oculomotor cranial nerve. It is the narrowing the pupil, which may be caused by scleral buckles or medicines such as opiates or anti hypertension medications. This phenomenon is calculated by dilation ratio [8].

Dilation Ratio - Dilation ratio is calculated as

$\Delta=$ Pupil radius/Iris radius

Few samples of dilated iris images is as show $\backslash n$ below 
a
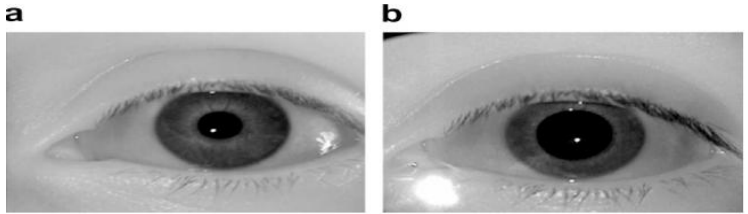

Fig 4: The iris image in part (a) was taken with an extra lamp in the room, and the image in (b) was taken with the subject wearing sunglasses. The pupil in (b) is almost as dilated (dilation ratio 0.54 ) as the most dilated pupil (0.56) seen in experimental data for this subject.

a

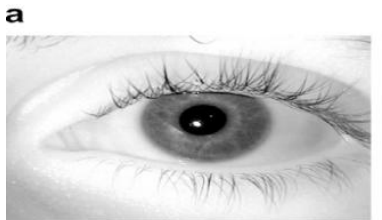

b

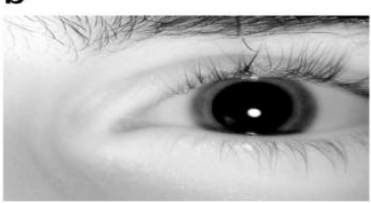

Fig 5: This subject showed the biggest difference in pupil size in the data set. The smallest dilation ratio (pupil radius/iris radius) for this subject was 0.3478 and the largest dilation ratio was $\mathbf{0 . 6 5 4 5}$.

a

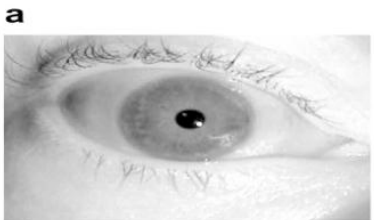

b

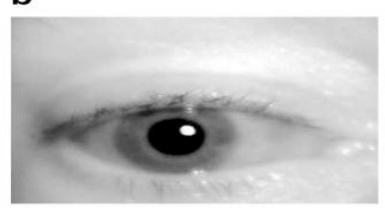

Fig 6: This subject had the smallest pupils in the data set. The smallest dilation ratio for this subject was 0.2137 , and the largest dilation ratio was 0.4762 .

\section{NORMALIZATION TECHNIQUES}

Considering case of no pupil dilation- After segmentation, the next phase is to normalize segmented portion in order to enable generation of the iris code and perform comparisons [9]. Since changes in the eye, like optical size of the iris, location of pupil in the iris, and the iris alignment changes from one person to another, it entails to normalize the iris image, so that the representation is familiar to all, with similar dimensions.

\subsection{Considering case on no pupil dilation}

Normalization process involves unfolding the iris and converting it into its polar counterpart. This is done using classic Daugman's Rubber sheet model $[9,10]$. The pivot of the pupil is considered as the threshold point and a remapping formula is utilized to convert the points on the cartesian [10] to the polar scale.

$\mathrm{r}^{\prime}=\sqrt{\alpha} \beta \pm \sqrt{ } \alpha \beta^{2}-\alpha-r_{1^{2}}$

Cartesian to polar conversion is as shown in the Figure 7 below

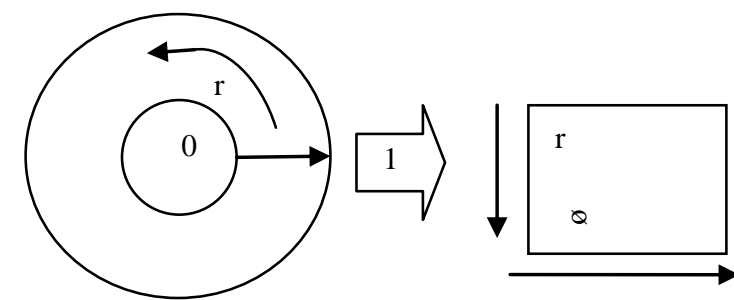

Fig 7: Cartesian to Polar conversion during Normalization

\subsection{Considering case of pupil dilation.}

The recent work of biomechanics can be used to approximate the nonlinear dynamics of the iris as a result of pupil dilation [11]. This approach accolades the classical pattern recognition principles with information about the muscle activity and the elastic properties of the iris. The mathematical analysis of the biomechanical model [12] begins by considering the iris region as a thin cylindrical shell. Here the $\mathrm{z}$ dimension is much smaller than the $r$ and $\theta$ dimensions, so the iris can be visualized as a thin plate where the loads are applied evenly over the $\mathrm{z}$ dimension

$\mathrm{r} 0=\mathrm{r}+\mathrm{u}(\mathrm{r})$

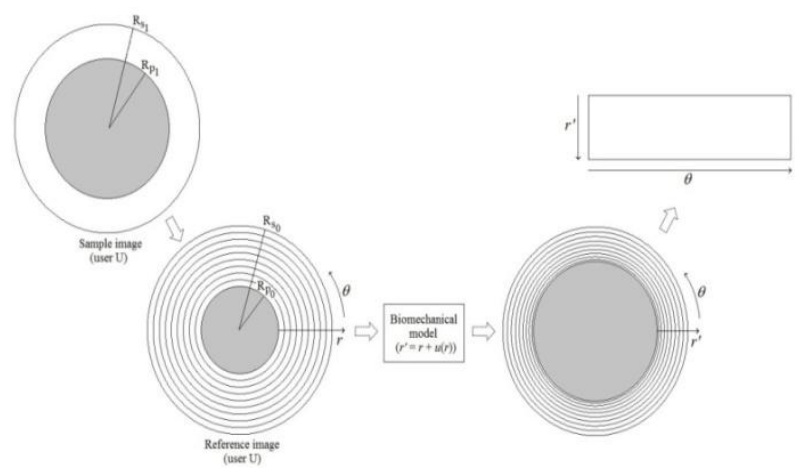

Fig 8: Cartesian to Polar conversion during Normalization in case of pupil dilation

Cartesian to polar conversion is as shown in the Figure 8 above.

\section{PROPOSED WORK}

In this paper a study of comparison of the effect of compression using JPEG 2000 compression [8] algorithm on normalized images samples received once through classic Daughman's rubber sheet model $[8,9]$ and the ones received through non linear biomechanical techniques for 2 different data set of each Normalization techniques is done. The performance accuracy will be determined by the comparison scores analyzed by the FAR and FRR scores.

This process is done in two steps which will be similar and repeated for each normalization techniques-

\subsection{Step 1: Simple cropping}

In this step there is reduction in image size from the standard iris image format of $320 \times 280$ pixels with 8 bits grayscale data per pixel resulting smaller size containing the iris as shown in Figure 7. The cropping is done manually to localize the iris as shown in Figure 8. 


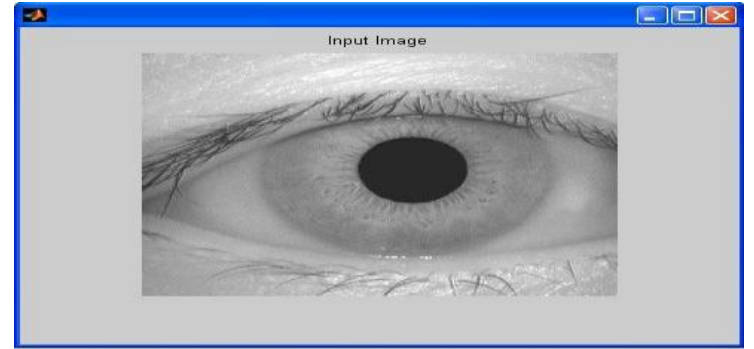

Fig 7: Input image sample

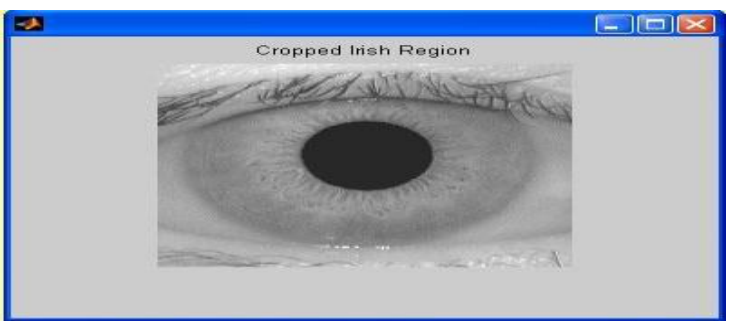

Fig 8: Cropped image sample

\subsection{Step 2: Finding Region of interest (ROI)}

The JPEG 2000 compression can be made more efficient if there is removal of all non-iris parts of the image with a uniform gray value. This kind of substitution of pixel values within a rectilinear image array is called finding region of interest or ROI [13]. The ROI image sample is as shown in the Figure 9 below

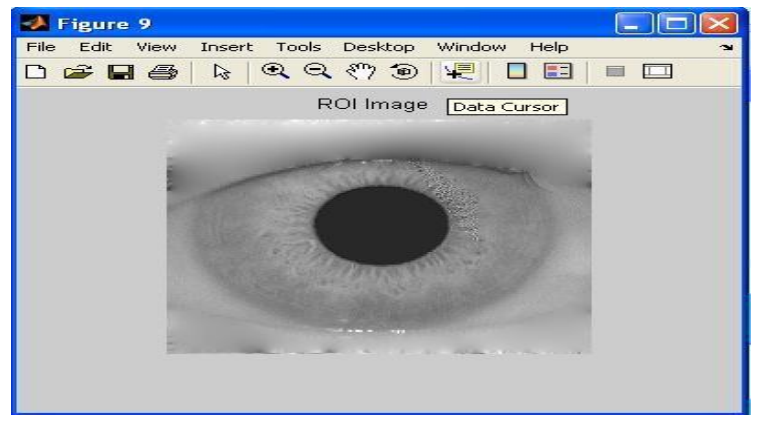

Fig 9: ROI image sample

\section{EXPERIMENTAL RESULTS}

Comparison of FAR and FRR using JPEG 2000 compression was done on 2 datasets for each of the normalization performed through classic Daughman's rubber sheet model and non linear biomechanical model [14]. The analysis was done as per Table 1 below

Table 1. FAR and FRR comparison using JPEG 2000 compression

\begin{tabular}{|l|l|l|l|}
\hline $\begin{array}{l}\text { Performance } \\
\text { metrics }\end{array}$ & Data set & $\begin{array}{l}\text { Non linear } \\
\text { Biomechanic } \\
\text { al model for } \\
\text { normalizatio } \\
\mathbf{n}\end{array}$ & $\begin{array}{l}\text { Daughman's } \\
\text { model for } \\
\text { normalization }\end{array}$ \\
\hline FAR & $\begin{array}{l}\text { Original } \\
\text { image }\end{array}$ & $\begin{array}{l}1 \text { in 500 at } \\
\text { FRR1 }\end{array}$ & $\begin{array}{l}1 \text { in } 500 \text { at } \\
\text { FRR1 }\end{array}$ \\
\cline { 2 - 5 } & CF $=25$ & $\begin{array}{l}1 \text { in 300 at } \\
\text { FRR1 in 200 at } \\
\text { FRR1 }\end{array}$ \\
\hline
\end{tabular}

\begin{tabular}{|l|l|l|ll|}
\hline & $\mathrm{CF}=35$ & $\begin{array}{l}1 \text { in 300 at } \\
\text { FRR1 }\end{array}$ & $\begin{array}{l}1 \text { in 200 at } \\
\text { FRR1 }\end{array}$ \\
\hline \multirow{4}{*}{ FRR } & $\mathrm{CF}=72$ & $\begin{array}{l}1 \text { in 10 at } \\
\text { FRR1 }\end{array}$ & $\begin{array}{l}\text { 1in 100 at } \\
\text { FRR1 }\end{array}$ \\
\hline & $\begin{array}{l}\text { Original } \\
\text { Image }\end{array}$ & 0.998 & 0.998 & \\
\hline & $\mathrm{CF}=25$ & 0.995 & 0.992 & \\
\hline & $\mathrm{CF}=35$ & 0.993 & 0.991 \\
\hline & $\mathrm{CF}=72$ & 0.991 & 0.988 \\
\hline
\end{tabular}

Biometric recognition performance is measured by ROC (Receiver Operating Characteristic) curves, which are a plot of the trade-off between two error rates i.e. False Accept Rate (FAR) and False Reject Rates(FRR). The decision threshold for similarity scores is varied from conventional to casual. The point at which the two error rates are equal, i.e. FRR $=$ FAR $=$ EER is called the Equal Error Rate. Also compression factor [15] or CF is calculated as

Compression factor $(\mathrm{CF})=$ un-compressed (original) image /compressed image

Figure 10 shows the ROC [16] curve drawn for JPEG 2000 compression.

The legend used in the experimental analysis is as depicted below

1. Green line shows the performance of original image,

2. Blue line shows the performance of compressed image at compression factor of 25 ,

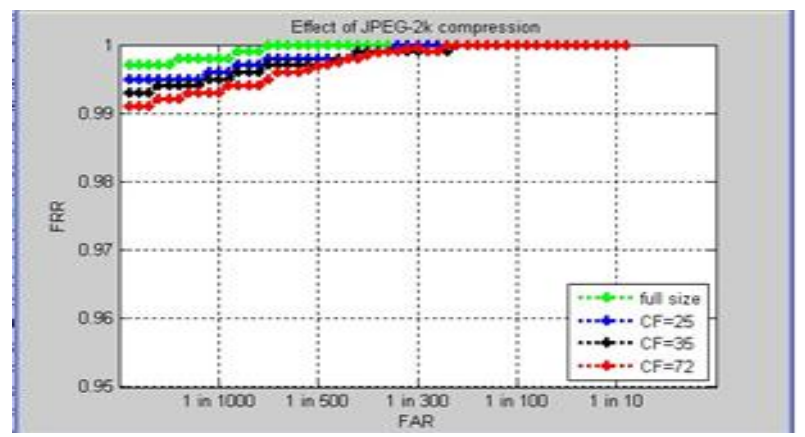

Fig 10: FAR and FRR plots in case of Daughman's normalization

3. Black line shows performance of compressed image at compression factor of $35 \&$

4. Red line shows performance of compressed image at compression factor of 72 .

It has been observed from ROC curve that recognition performance is improved if JPEG 2000 technique is used for images samples normalized with non linear bio mechanical model to that done through classic Daughman's model.

Also an additional experimental analysis is done for the PSNR [17] for various compression algorithms as shown in the Figure 11 below 


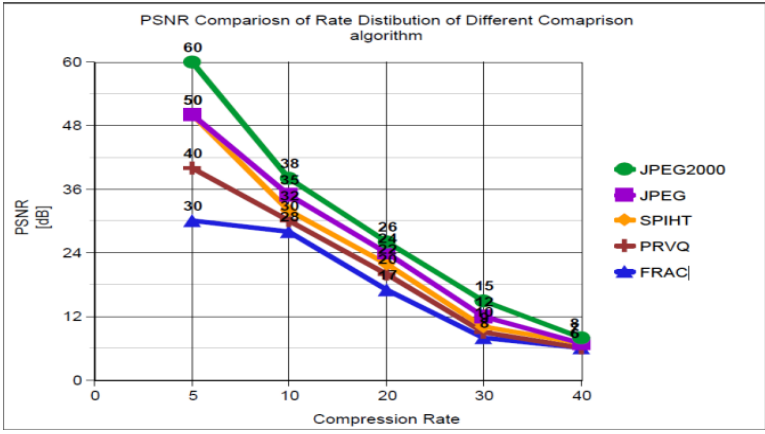

Fig 11: PSNR comparison for various Compression algorithms

The following set of data was used for this comparison. This comparison was repeated for Hamming distance [18].

\subsection{HD=0 i.e. Legitimate user or correct match}

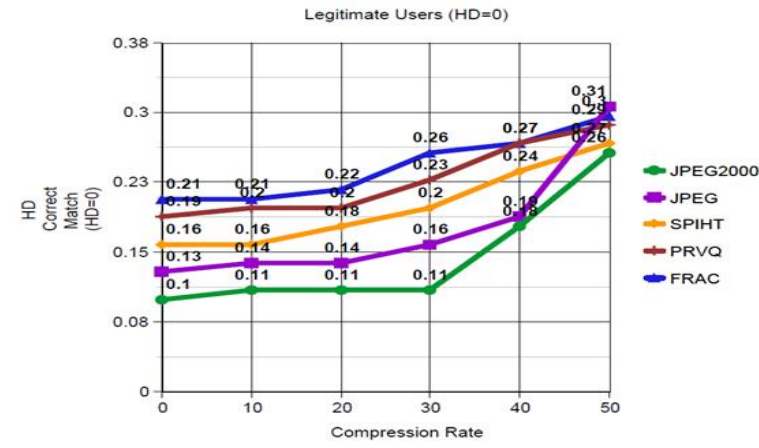

Fig 12: HD=0 Perfect Match case for Proposed JPEG 2000 algorithm

\subsection{HD=0.5 i.e. Imposter user or correct mismatch}

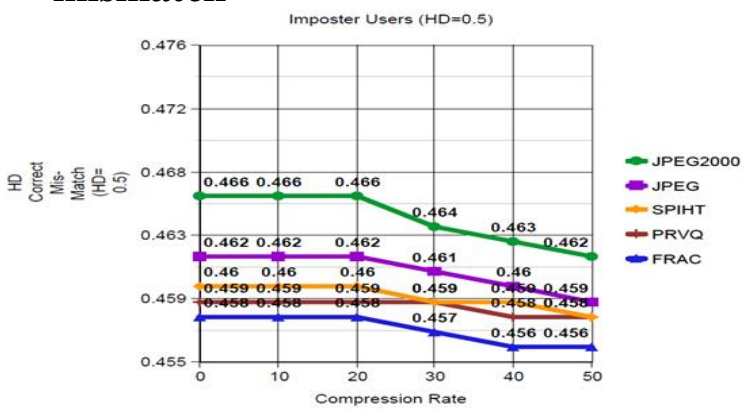

Fig 13: HD=0.5 Perfect MIS- Match case for Proposed JPEG 2000 algorithm

As per the above analysis it is observed that the optimized JPEG 2000 algorithm shows better results by a factor of 20-30 $\%$ better results achieved with the optimized JPEG 2000 algorithm. Also the optimization performs better in terms of speed of comparison and memory management of the stored iris templates $[19,20]$.

\section{CONCLUSION AND FUTURE WORK}

In this paper analysis of image compression on iris recognition performance by using JPEG 2000 compression algorithm is studied. It has been observed that as compared to the classic method of normalization using Daughman's algorithm, the effect of compression yields better results in terms of FAR and FRR performance metrics for the normalization technique used for the case of pupil dilation employing non linear biomechanical. Out of 2 normalization methods used the JPEG 2000 compression schemes used gives the best performance in term of ROC curve for non linear biomechanical model of normalization. It is concluded that image compression has less severe effect over image acquired and normalized through non linear biomechanical models [20], inspite of employing classic methods of normalization like Daughman's model [8].

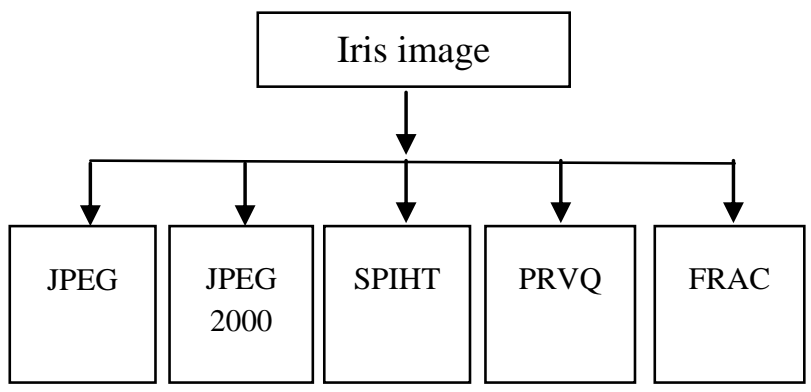

Fig 14: Classifications of Iris Compression Methods

As depicted in Figure 14 and as a future scope there is a plan for employing the various compression algorithms over iris recognition methods used currently and study the performance of compression over various samples to analysis the effect of compression over images samples during matching stages without compromising the recognition performance accuracy.

\section{REFERENCES}

[1] I.J Shaikh, S.M Mukane, The Effect of Iris Image Compression on Recognition Performance published in International Journal of Computer Applications (0975 8887) Volume 113 - No. 18, March 2015

[2] A.P. Bradley and F.W.M. Stentiford, "JPEG2000 and region of interest coding," Digital Imaging Computing Techniques and Applications, Melbourne, 2002. Available at: http://www.ee.ucl.ac.uk/ fstentif/DICTA02.p

[3] Brian C. Smith, Lawrence A. Rowe, Compressed Domain Processing of JPEG-encoded images.pdf,International Journal of Computer Applications (0975 - 8887) Volume 65 - No. 04, September 2015

[4] MajidRabbani, Rajan Joshi, An overview of the JPEG2000 still image compression standard, Signal Processing: Image Communication 17 (2002) 348,Elsevier volume 48, 2014

[5] Robert Buckley, Using Lossy JPEG 2000 Compression For Archival Master Files.pdf, Prime Contract/Solicitation No.: LCOSI10T0057 Prime Task Order No. OSI12T0010, March, 2013

[6] Michael W. Marcellin, Michael J. Gormish, Ali Bilgin1, Martin P. Boliek, An Overview of JPEG-2000.pdf,IEEE Data Compression Conference, pp. 523-541, 2015

[7] RituChourasiya and Prof. AjitShrivastava, a study of image compression based transmission algorithm using spihtforlow bit rate application.pdf, Advanced Computing: An International Journal ( ACIJ ), Vol.3, No.6, November 2012 
[8] SwethaDodla, Y David SolmonRaju, K V Murali Mohan, Image Compression using Wavelet and SPIHT Encoding Scheme, International Journal of Engineering Trends and Technology (IJETT) - Volume 4 Issue 9- Sep 2013

[9] Mascher-Kampfer, Herbert St"ogner, and Andreas Uhl1,Comparison of Compression Algorithms' Impact on Fingerprint and Face Recognition Accuracy. pdf, International Journal of Engineering Trends and Technology (IJETT) - Volume 4 Issue 9- Sep 2015

[10] Hannes Hartenstein, Associate Member, IEEE, Matthias Ruhl, and DietmarSaupe, Region-Based Fractal Image Compression, IEEE TRANSACTIONS ON IMAGE PROCESSING, VOL. 9, NO. 7, JULY 2015

[11] Alphones Maria, Amlu Anna Joshy, Aneeta Christopher, Anjali B4, RanjithaRajan, Iris Database Compression Using Haar Wavelet Decomposition and Huffman Coding, International Journal of Advanced Research in Computer and Communication Engineering Vol. 5, Issue 4, April 2016

[12] MayankVatsa , Richa Singh, P .Gupta, Improving Iris Recognition Performance Using Segmentation, Quality Enhancement, Match Score Fusion, and Indexing

[13] A.K.Jain,A.Ross, andS.Pankanti,"Biometrics:ATool for Information Security", IEEE Transactions on InformationForensicsandSecurity,Vol.1,No.2,2006,pp. 125-143.

[14] V.Dorairaj,A. Schmid, and G. Fahmy,"Performance Evaluation of Iris Based
RecognitionSystemImplementing

PCAandICAEncodingTechniques",inProceedings

of SPIE,2005,pp.51-58.

[15] S.Shah,andA.Ross,"IrisSegmentationUsingGeodesic Active Contours", IEEE Trans. InformationForensics andSecurity,Vol.4,No.4,2009,pp.824-836.

[16] I.JShaikh, S.M Mukane, The Effect of Iris Image Compression on Recognition Performance published in International Journal of Computer Applications (0975 8887) Volume 113 - No. 18, March 2015

[17] A.P. Bradley and F.W.M. Stentiford, "JPEG2000 and region of interest coding," Digital Imaging ComputingTechniques and Applications, Melbourne, 2002. Available at: http://www.ee.ucl.ac.uk/fstentif/DICTA02.p

[18] Surjeet Singh, Kulbir Singh, Segmentation Techniques for Iris Recognition System,Iinternational Journal of Scientific \& Engineering Res V volume 2, Issue 4, April

[19] J. Daugman. How iris recognition works. Proceedings of nternational Conference on Image Processing, Vol. 1, 2002.

[20] A.M.Raid, W.M.Khedr, M. A. El-dosuky and Wesam Ahmed, Jpeg Image Compression Using Discrete CosineTransform.pdf - A Survey, International Journal of Computer Science \& Engineering Survey (IJCSES) Vol.5, No.2, April 2014 